\title{
On a Fuzzy Problem with Variable Coefficient by Fuzzy Laplace Transform
}

\section{Hülya GÜLTEKİN ÇİTİ ${ }^{1 *}$}

ABSTRACT: This study is on solutions of a fuzzy problem with variable coefficient. Solutions are found by fuzzy Laplace transform. Generalized differentiability is used. It is searched whether the solutions are valid $\alpha$-level sets or not. Examples are solved on studied problem. Conclusions are given. Keywords: Fuzzy initial value problem, fuzzy Laplace transform, generalized differentiability, triangular fuzzy number

1 Hülya GÜLTEKİN ÇíTíl (Orcid ID: 0000-0002-3543-033X), Department of Mathematics, Faculty of Arts and Sciences, Giresun University, Giresun, Turkey

*Sorumlu Yazar/Corresponding Author: Hülya GÜLTEKİN ÇİTİL, e-mail: hulya.citil@giresun.edu.tr 


\section{INTRODUCTION}

The theory of fuzzy differential equation is very important topic. Therefore, it is studied by many researchers. For example, in population models (Buckley et al., 2002) and growth model (Mondal at al., 2013).

Zadeh first introduced fuzzy number and fuzzy arithmetic (Zadeh, 1965). There are different approaches to solve the fuzzy differential equation. The first approach is Hukuhara derivative (Puri and Ralescu, 1983; Gültekin and Altınışık, 2014) or generalized Hukuhara derivative (Stefanini and Bede 2008; Ceylan and Altınışık, 2018; Gültekin Çitil, 2018). But in Hukuhara derivative, the solution becomes uncertain as time goes on. Thus, generalized Hukuhara derivative was introduced (Bede at al., 2007). The second approach is extension principle (Zadeh, 1975). The third approach is differential inclusion (Hüllermeier, 1997).

Fuzzy Laplace transform is important topic to solve fuzzy differential equation. Also, it gives solution satisfying the initial values of fuzzy differential equation directly. Firstly, it was introduced by Allahviranloo and Barchordary Ahmadi (2010). They solved first order fuzzy initial value problem by fuzzy Laplace transform. In many areas, many researchers used fuzzy Laplace transform (Ramazannia Tolouti and Barchordary Ahmadi, 2010; Ahmad at al., 2012; Mondal and Roy, 2015).

In this paper, a fuzzy problem with variable coefficient is investigated by fuzzy Laplace transform. The concept of generalized differentiability, the properties of fuzzy Laplace transform, fuzzy arithmetic, Hukuhara difference are used. It is searched whether the solutions are valid fuzzy functions or not. Examples are solved.

In section 2 , it is given definitions and theorems that we will use in our study. In section 3 , the considered problem is introduced and the problem is solved by fuzzy Laplace transform, several theorems are given and examples are solved. In section 4, conclusion is given.

\section{MATERIALS AND METHODS}

Definition 1. A fuzzy number is a mapping $u: \mathbb{R} \rightarrow[0,1]$ where,

$\overline{\{x \in \mathbb{R} \mid u(x)>0\}}$ is compact, $u$ is convex fuzzy set, normal, upper semi-continuous on $\mathbb{R}$ (Liu, 2011).

Let's show the set of all fuzzy numbers with $\mathbb{R}_{F}$.

Definition 2. $u \in \mathbb{R}_{F},[u]^{\alpha}=\{x \in \mathbb{R} \mid u(x) \geq \alpha\}=\left[\underline{u}_{\alpha}, \bar{u}_{\alpha}\right], 0<\alpha \leq 1$ is the $\alpha$-level set of $u$.

If $\alpha=0,[u]^{0}=\overline{\{\text { suppu }\}}=\overline{\{x \in \mathbb{R} \mid u(x)>0\}}$ (Khastan and Nieto, 2010).

Definition 3. The parametric form $\left[\underline{u}_{\alpha}, \bar{u}_{\alpha}\right]$ of A fuzzy number satisfty the following requirements:

The lower part $\underline{u}_{\alpha}$ is left-continuous bounded non-decreasing on $(0,1]$, also it is rightcontinuous for $\alpha=0$,

The upper part $\bar{u}_{\alpha}$ is left-continuous bounded non-increasing on $(0,1]$, also it is rightcontinuous for $\alpha=0$,

$\underline{u}_{\alpha} \leq \bar{u}_{\alpha}, 0 \leq \alpha \leq 1$ (Salahshour and Allahviranloo, 2013). 
Definition 4. $[B]^{\alpha}=\left[\underline{b}+\left(\frac{\bar{b}-\underline{b}}{2}\right) \alpha, \bar{b}-\left(\frac{\bar{b}-\underline{b}}{2}\right) \alpha\right]$ is the $\alpha$-level set of $B$, which is a symmetric triangular fuzzy number with support $[\underline{b}, \bar{b}]$ (Liu, 2011).

Definition 5. The metric is defined by

$D(u, v)=\sup _{0 \leq \alpha \leq 1} d\left([u]^{\alpha},[v]^{\alpha}\right)$,

$d\left([u]^{\alpha},[v]^{\alpha}\right)=\max \left\{\left|\underline{u}_{\alpha}-\underline{v}_{\alpha}\right|,\left|\bar{u}_{\alpha}-\bar{v}_{\alpha}\right|\right\}$

on $\mathbb{R}_{F}$ (Fatullayev at al., 2013).

Definition 6. Let $u, v \in \mathbb{R}_{F}$. If there exists $w \in \mathbb{R}_{F}$ such that $u=v+w, w$ is Hukuhara difference of $u$ and $v, w=u \ominus v$ (Puri and Ralescu, 1983).

Definition 7. Let $f:[a, b] \rightarrow \mathbb{R}_{F}$ and $x_{0} \in[a, b]$. If there exists $f^{\prime}\left(x_{0}\right) \in \mathbb{R}_{F}$ such that for all $h>0$ sufficiently small, $\exists f\left(x_{0}+h\right) \ominus f\left(x_{0}\right), f\left(x_{0}\right) \ominus f\left(x_{0}-h\right)$ and the limits hold

$\lim _{h \rightarrow 0} \frac{f\left(x_{0}+h\right) \ominus f\left(x_{0}\right)}{h}=\lim _{h \rightarrow 0} \frac{f\left(x_{0}\right) \ominus f\left(x_{0}-h\right)}{h}=f^{\prime}\left(x_{0}\right)$,

$f$ is Hukuhara differentiable at $x_{0}$ (Bede, 2008).

Definition 8. Let $f:[a, b] \rightarrow \mathbb{R}_{F}$ and $x_{0} \in[a, b]$. If there exists $f^{\prime}\left(x_{0}\right) \in \mathbb{R}_{F}$ such that for all $h>0$ sufficiently small, $\exists f\left(x_{0}+h\right) \ominus f\left(x_{0}\right), f\left(x_{0}\right) \ominus f\left(x_{0}-h\right)$ and the limits hold

$\lim _{h \rightarrow 0} \frac{f\left(x_{0}+h\right) \ominus f\left(x_{0}\right)}{h}=\lim _{h \rightarrow 0} \frac{f\left(x_{0}\right) \ominus f\left(x_{0}-h\right)}{h}=f^{\prime}\left(x_{0}\right)$,

$f$ is (1)-differentiable at $x_{0}$. If there exists $f^{\prime}\left(x_{0}\right) \in \mathbb{R}_{F}$ such that for all $h>0$ sufficiently small, $\exists f\left(x_{0}\right) \ominus f\left(x_{0}+h\right), f\left(x_{0}-h\right) \ominus f\left(x_{0}\right)$ and the limits hold

$\lim _{h \rightarrow 0} \frac{f\left(x_{0}\right) \ominus f\left(x_{0}+h\right)}{-h}=\lim _{h \rightarrow 0} \frac{f\left(x_{0}-h\right) \ominus f\left(x_{0}\right)}{-h}=f^{\prime}\left(x_{0}\right)$,

$f$ is (2)-differentiable at $x_{0}$. (Khastan and Nieto, 2010).

Theorem 1. Let $f:[a, b] \rightarrow \mathbb{R}_{F}$ be a function and denote $[f(t)]^{\alpha}=\left[\underline{f}_{\alpha}(t), \bar{f}_{\alpha}(t)\right]$ for each $\alpha \in[0,1]$.

1. If the function $f$ is (1)-differentiable, the lower function $\underline{f}_{\alpha}(t)$ and the upper function $\bar{f}_{\alpha}(t)$ are differentiable,

$\left[f^{\prime}(t)\right]^{\alpha}=\left[\underline{f}_{\alpha}^{\prime}(t), \bar{f}_{\alpha}^{\prime}(t)\right]$.

2. If the function $f$ is (2)-differentiable, the lower function $\underline{f}_{\alpha}(t)$ and the upper function $\bar{f}_{\alpha}(t)$ are differentiable,

$\left[f^{\prime}(t)\right]^{\alpha}=\left[\bar{f}_{\alpha}^{\prime}(t), \underline{f}_{\alpha}^{\prime}(t)\right] \quad($ Khastan at al., 2009). 
Definition 9. Let $f:[a, b] \rightarrow \mathbb{R}_{F}$ be a fuzzy function. The fuzzy Laplace transform of $f$ is

$F(s)=L(f(t))=\int_{0}^{\infty} e^{-s t} f(t) d t=\left[\lim _{\rho \rightarrow \infty} \int_{0}^{\rho} e^{-s t} \underline{f}(t) d t, \lim _{\rho \rightarrow \infty} \int_{0}^{\rho} e^{-s t} \bar{f}(t) d t\right]$.

$F(s, \alpha)=L\left([f(t)]^{\alpha}\right)=\left[L\left(\underline{f}_{\alpha}(t)\right), L\left(\bar{f}_{\alpha}(t)\right)\right]$,

$L\left(\underline{f_{\alpha}}(t)\right)=\int_{0}^{\infty} e^{-s t} \underline{f_{\alpha}}(t) d t=\lim _{\rho \rightarrow \infty} \int_{0}^{\rho} e^{-s t} \underline{f_{\alpha}}(t) d t$,

$L\left(\bar{f}_{\alpha}(t)\right)=\int_{0}^{\infty} e^{-s t} \bar{f}_{\alpha}(t) d t=\lim _{\rho \rightarrow \infty} \int_{0}^{\rho} e^{-s t} \bar{f}_{\alpha}(t) d t$ (Patel and Desai, 2017).

Theorem 2. Let $f^{\prime}(t)$ be an integrable fuzzy function, $f(t)$ is primitive of $f^{\prime}(t)$ on $(0, \infty]$. If $f$ is (1)differentiable,

$L\left(f^{\prime}(t)\right)=s L(f(t)) \ominus f(0)$,

if $f$ is (2)-differentiable,

$L\left(f^{\prime}(t)\right)=(-f(0)) \ominus(-s L(f(t)))$ (Allahviranloo and Barchordari Ahmadi, 2010).

Theorem 3. Let be $f(x), g(x)$ continuous fuzzy functions and $c_{1}, c_{2}$ constants, then

$L\left(\left(c_{1} \odot f(x)\right) \oplus\left(c_{2} \odot g(x)\right)\right)=\left(c_{1} \odot L(f(x))\right) \oplus\left(c_{2} \odot L(g(x))\right) \quad$ (Allahviranloo and Barchordari Ahmadi, 2010).

Theorem 4. Let $f^{\prime \prime}(t)$ be an integrable fuzzy function and $f(t), f^{\prime}(t)$ are primitive of $f^{\prime}(t), f^{\prime \prime}(t)$ on $(0, \infty]$. Then, if $f$ and $f^{\prime}$ are (1)-differentiable,

$L\left(f^{\prime \prime}(t)\right)=s^{2} L(f(t)) \ominus s f(0) \ominus f^{\prime}(0)$,

if $f$ and $f^{\prime}$ are (2)-differentiable,

$L\left(f^{\prime \prime}(t)\right)=s^{2} L(f(t)) \ominus s f(0)-f^{\prime}(0)$,

if $f$ is (1)-differentiable and $f^{\prime}$ is (2)-differentiable,

$L\left(f^{\prime \prime}(t)\right)=\ominus\left(-s^{2}\right) L(f(t))-s f(0)-f^{\prime}(0)$

if $f$ is (2)-differentiable and $f^{\prime}$ is (1)-differentiable,

$L\left(f^{\prime \prime}(t)\right)=\ominus\left(-s^{2}\right) L(f(t))-s f(0) \ominus f^{\prime}(0)$ (Patel and Desai, 2017).

Theorem 5. Let $f(t)$ satisfies the condition of existence theorem of Laplace transform and

$L(f(t))=F(s)$,

then

$L(t f(t))=-F^{\prime}(s)$.

If $f^{\prime}(t)$ satisfies the condition of existence theorem of Laplace transform, then

$L\left(t f^{\prime}(t)\right)=-s F^{\prime}(s)-F(s)$,

similarly for $f^{\prime \prime}(t)$

$L\left(t f^{\prime \prime}(t)\right)=-s^{2} F^{\prime}(s)-2 s F(s)+f(0)$ (Patel and Desai, 2017). 


\section{RESULTS AND DISCUSSION}

Consider fuzzy problem with variable coefficient

$$
t y^{\prime \prime}+\lambda y^{\prime}=[A]^{\alpha}, y(0)=[B]^{\alpha}, t>0
$$

by fuzzy Laplace transform, where $\lambda<0$,

$$
\begin{aligned}
& {[A]^{\alpha}=\left[\underline{A}_{\alpha}, \bar{A}_{\alpha}\right]=\left[\underline{a}+\left(\frac{\bar{a}-\underline{a}}{2}\right) \alpha, \bar{a}-\left(\frac{\bar{a}-\underline{a}}{2}\right) \alpha\right],} \\
& {[B]^{\alpha}=\left[\underline{B}_{\alpha}, \bar{B}_{\alpha}\right]=\left[\underline{b}+\left(\frac{\bar{b}-\underline{b}}{2}\right) \alpha, \bar{b}-\left(\frac{\bar{b}-\underline{b}}{2}\right) \alpha\right]}
\end{aligned}
$$

are symmetric triangular fuzzy numbers with the supports $[\underline{a}, \bar{a}],[\underline{b}, \bar{b}]$, respectively.

Firstly, taking the Laplace transform of fuzzy differential equation (1), we have

$L\left(t y^{\prime \prime}+\lambda y^{\prime}\right)=L\left([A]^{\alpha}\right)$.

Using the properties of fuzzy Laplace transform,

$$
L\left(t y^{\prime \prime}\right)+\lambda L\left(y^{\prime}\right)=L\left([A]^{\alpha}\right) \text {. }
$$

Using the Theorem 4 and Theorem 5, we obtain

$$
-s^{2} Y^{\prime}(s, \alpha)-2 s Y(s, \alpha)-(\ominus y(0, \alpha))+\lambda(s Y(s, \alpha) \ominus y(0, \alpha))=L\left([A]^{\alpha}\right) .
$$

Using the Hukuhara difference, fuzzy arithmetic and the properties of fuzzy Laplace transform, the equations

$$
\begin{aligned}
& -s^{2} \underline{Y}^{\prime}(s, \alpha)-2 s \underline{Y}(s, \alpha)+\underline{y}(0, \alpha)+\lambda s \underline{Y}(s, \alpha)-\lambda \underline{y}(0, \alpha)=\frac{\bar{A}_{\alpha}}{s}, \\
& -s^{2} \bar{Y}^{\prime}(s, \alpha)-2 s \bar{Y}(s, \alpha)+\bar{y}(0, \alpha)+\lambda s \bar{Y}(s, \alpha)-\lambda \bar{y}(0, \alpha)=\frac{\underline{A}_{\alpha}}{s}
\end{aligned}
$$

are obtained. The equation (5) gives the equation

$$
\underline{Y}^{\prime}(s, \alpha)+\frac{2-\lambda}{s} \underline{Y}(s, \alpha)=-\frac{\bar{A}_{\alpha}}{s^{3}}+\frac{(1-\lambda) \underline{B}_{\alpha}}{s^{2}} .
$$

Using the integral multiplier

$$
\begin{aligned}
& \mu(s)=e^{\int \frac{2-\lambda}{s} d s}=s^{2-\lambda}, \\
& s^{2-\lambda} \underline{Y}(s, \alpha)=\int\left(-\frac{\bar{A}_{\alpha}}{s^{1+\lambda}}+\frac{(1-\lambda) \underline{B_{\alpha}}}{s^{\lambda}}\right) d s
\end{aligned}
$$

is obtained. From this, we have

$$
\underline{Y}(s, \alpha)=\frac{\bar{A}_{\alpha}}{\lambda} \frac{1}{s^{2}}+\underline{B}_{\alpha} \frac{1}{s}+C \frac{1}{s^{2-\lambda}} .
$$

Taking the inverse Laplace transform of (10), the lower solution of the problem (1) is obtained as

$$
\underline{y}(t, \alpha)=\frac{\bar{A}_{\alpha}}{\lambda} t+\underline{B}_{\alpha}+C_{*} t^{1-\lambda} .
$$

Similarly, the upper solution of the problem (1) is obtained as

$$
\bar{y}(t, \alpha)=\frac{A_{\alpha}}{\lambda} t+\bar{B}_{\alpha}+C_{*} t^{1-\lambda}
$$


Consequently, the solution of the problem (1) is

$$
[y(t)]^{\alpha}=[\underline{y}(t, \alpha), \bar{y}(t, \alpha)]
$$

by fuzzy Laplace transform.

Now let's investigate whether the solution is a valid fuzzy function or not.

Theorem 1. The solution $[y(t)]^{\alpha}=[\underline{y}(t, \alpha), \bar{y}(t, \alpha)]$ of the problem (1) is a valid $\alpha$-level set.

Proof. As

$\frac{\partial \underline{y}(t, \alpha)}{\partial \alpha}>0, \frac{\partial \bar{y}(t, \alpha)}{\partial \alpha}<0, \underline{y}(t, \alpha)<\bar{y}(t, \alpha)$,

the solution of the problem (1) is a valid $\alpha$-level set. According to this, it must be $-\frac{t}{\lambda}\left(\frac{\bar{a}-\underline{a}}{2}\right)+\left(\frac{\bar{b}-\underline{b}}{2}\right)>0$.

That is, $t>\frac{\lambda(\bar{b}-\underline{b})}{(\bar{a}-\underline{a})}$.

Since $\bar{b}-\underline{b}>0, \bar{a}-\underline{a}>0$ and $\lambda<0$, this equality is provided. The proof is completed.

Theorem 2. The solution $[y(t)]^{\alpha}=[\underline{y}(t, \alpha), \bar{y}(t, \alpha)]$ of the problem (1) is symmetric triangular fuzzy number for any $t>0$ time.

Proof. Since

$\underline{y}(t, 1)=\frac{t}{\lambda}\left(\frac{\bar{a}+\underline{a}}{2}\right)+\left(\frac{\bar{b}+\underline{b}}{2}\right)+C_{*} t^{1-\lambda}=\bar{y}(t, 1)$

and

$\underline{y}(t, 1)-\underline{y}(t, \alpha)=(1-\alpha)\left(\left(\frac{\bar{b}-\underline{b}}{2}\right)-\frac{t}{\lambda}\left(\frac{\bar{a}-\underline{a}}{2}\right)\right)=\bar{y}(t, \alpha)-\bar{y}(t, 1)$,

the solution of the problem (1) is symmetric triangular fuzzy number for any $t>0$ time

Example 1. We consider the fuzzy problem

$t y^{\prime \prime}-y^{\prime}=[0]^{\alpha}, y(0)=[1]^{\alpha}$

by the fuzzy Laplace transform, where $[0]^{\alpha}=[-1+\alpha, 1-\alpha],[1]^{\alpha}=[\alpha, 2-\alpha]$.

Solving the fuzzy problem (14), the solution is obtained as

$\underline{y}(t, \alpha)=\alpha+(\alpha-1) t+C_{*} t^{2}$,

$\bar{y}(t, \alpha)=(2-\alpha)+(1-\alpha) t+C_{*} t^{2}$,

$[y(t)]^{\alpha}=[\underline{y}(t, \alpha), \bar{y}(t, \alpha)]$.

The solution (15)-(17) of the problem (14) is a valid $\alpha$-level set since $t>-1$ and the solution is symmetric triangular fuzzy number for any $t>0$ time. 
Example 2. Consider the problem

$t y^{\prime \prime}-2 y^{\prime}=[1]^{\alpha}, y(0)=[2]^{\alpha}$

where $[1]^{\alpha}=[\alpha, 2-\alpha],[2]^{\alpha}=[1+\alpha, 3-\alpha]$.

Solving the problem (18) by fuzzy Laplace transform, we obtain

$\underline{y}(t, \alpha)=(1+\alpha)+\left(\frac{\alpha}{2}-1\right) t+C_{*} t^{2}$,

$\bar{y}(t, \alpha)=(3-\alpha)-\frac{\alpha}{2} t+C_{*} t^{2}$,

$[y(t)]^{\alpha}=[\underline{y}(t, \alpha), \bar{y}(t, \alpha)]$.

The solution (19)-(21) of the problem (18) is a valid $\alpha$-level set since $t>-2$ and the solution is symmetric triangular fuzzy number for any $t>0$ time.

\section{CONCLUSION}

In this paper, a fuzzy problem with variable coefficient and symmetric triangular fuzzy number initial value is searched by fuzzy Laplace transform. It is used the concept of generalized differentiability. It is found that the solution is a valid $\alpha$-level set. Also, it is found that the solution is symmetric triangular fuzzy number for any $t>0$ time. Different problems can be investigated and different conclusions can be found. This topic is important and it will be useful for other mathematicians.

\section{REFERENCES}

Ahmad N, Mamat M, Kavikumar J, Amir Hamzah NS, 2012. Solving fuzzy duffing's equation by the fuzzy Laplace transform decomposition. Applied Mathematical Sciences, 6(59):2935-2944.

Allahviranloo T, Barkhordari Ahmadi M, 2010. Fuzzy Laplace transforms, Soft Computing, 14(3):235243.

Bede B, 2008. Note on "Numerical solutions of fuzzy differential equations by predictor-corrector method'. Information Sciences, 178(7):1917-1922.

Bede B, Rudas IJ, Bencsik AL, 2007. First order linear fuzzy differential equations under generalized differentiability. Information Sciences, 177(7):1648-1662.

Buckley JJ, Feuring T, Hayashi Y, 2002. Linear systems of first order ordinary differential equations: Fuzzy initial conditions. Soft Computing, 6(6):415-421.

Ceylan T, Altınışık N, 2018. Fuzzy eigenvalue problem with eigenvalue parameter contained in the boundary condition. Journal of Science and Arts, 3(44):589-602.

Fatullayev AG, Can E, Köroğlu C, 2013. Numerical solution of a boundary value problem for a second order fuzzy differential equation, TWMS Journal of Pure and Applied Mathematics, 4(2):169-176.

Gültekin H, Altınışık N, 2014. On solution of two-point fuzzy boundary value problems. The Bulletin of Society for Mathematical Services and Standards, 11:31-39.

Gültekin Çitil H, 2018. The relationship between the solutions according to the noniterative method and the generalized differentiability of the fuzzy boundary value problem. Malaya Journal of Matematik, 6(4):781-787. 
Hüllermeier E, 1997. An approach to modelling and simulation of uncertain dynamical systems. International Journal of Uncertainty, Fuzziness and Knowledge-Based Systems, 5(2):117-137.

Khastan A, Bahrami F, Ivaz K, 2009. New results on multiple solutions for nth-order fuzzy differential equations under generalized differentiability. Boundary Value Problems, doi:10.1155/2009/395714, $1-13$.

Khastan A, Nieto JJ, 2010. A boundary value problem for second order fuzzy differential equations. Nonlinear Analysis, 72(9-10):3583-3593.

Liu H-K, 2011. Comparison results of two-point fuzzy boundary value problems. International Journal of Computational and Mathematical Sciences, 5(1):1-7.

Mondal SP, Banerjee S, Roy TK, 2013. First order linear homogeneous ordinary differential equation in fuzzy environment. International Journal of Pure and Applied Sciences and Technology, 14(1):16-26.

Mondal SP, Roy TK, 2015. Generalized intuitionistic fuzzy Laplace transform and its application in electrical circuit. TWMS Journal of Applied and Engineering Mathematics, 5(1):30-45.

Patel KR, Desai NB, 2017. Solution of variable coefficient fuzzy differential equations by fuzzy Laplace transform. International Journal on Recent and Innovation Trends in Computing and Communication, 5(6):927-942.

Puri ML, Ralescu DA, 1983. Differentials of fuzzy functions. Journal of Mathematical Analysis and Applications, 91(2):552-558.

Ramazannia Tolouti SJ, Barkhordary Ahmadi M, 2010. Fuzzy Laplace transform on two order derivative and solving fuzzy two order differential equations. International Journal of Industrial Mathematics, 4(2):279-293.

Salahshour S. Allahviranloo T, 2013. Applications of fuzzy Laplace transforms. Soft Computing, 17(1):145-158.

Stefanini L, Bede B, 2008. Generalized Hukuhara differentiability of interval-valued functions and interval differential equations. Nonlinear Analysis, 71(3-4):1311-1328.

Zadeh LA, 1965. Fuzzy sets. Information and Control, 8(3):338-353.

Zadeh LA, 1975. The concept of a linguistic variable and its application to approximate reasoning-I. Information Sciences, 8(3):199-249. 